\title{
Paradoxical fluorescence after administration of 5-aminolevulinic acid for resection of a cerebral melanoma metastasis
}

\author{
Philippe Schucht • Jürgen Beck • Istvan Vajtai • \\ Andreas Raabe
}

Received: 16 February 2011 /Accepted: 2 March 2011 /Published online: 24 May 2011

(C) Springer-Verlag 2011

Sir,

Five-aminolevulinic acid (5-ALA) is increasingly used for resection of high-grade glioma; however, little is known of its fluorescent behavior in other cerebral tumors. In this letter, we report our findings of a paradoxical fluorescent pattern after administration of 5-ALA in a metastatic melanoma.

A 69-year-old man was admitted for apraxia and disorientation after a skiing accident. The patient's previous medical history included the excision of malignant melanomas 9 years earlier, with no known metastatic spread. Magnetic resonance imaging (MRI) showed a brightly enhancing, centrally necrotic, solitary right frontal tumor mass, indicating a glioblastoma or metastasis.

A solution of $1.5 \mathrm{~g}$ ALA was orally administered $4 \mathrm{~h}$ preoperatively. A craniotomy guided by neuronavigation was performed, followed by durotomy. Using a surgical microscope and light source at 5-ALA excitation wavelength (OPMI Neuro/NC4 system-fluorescence kit, Carl Zeiss, Oberkochen, Germany), neither bright nor vague fluorescence

P. Schucht $(\square) \cdot$ J. Beck $\cdot$ A. Raabe

Department of Neurosurgery, Bern University Hospital,

Inselspital,

3010 Bern, Switzerland

e-mail: philippe.schucht@insel.ch

I. Vajtai

Department of Neuropathology, Bern University Hospital, Inselspital,

Bern, Switzerland was emitted by the tumor mass. The surrounding tissue, however, exhibited solid red fluorescence up to a distance of $5 \mathrm{~mm}$ from the solid tumor core. The intraoperative navigation system (Brainlab, Feldkirchen, Germany) confirmed that the peritumoral "5-ALA-positive" fluorescent tissue was outside the strongly enhancing tumor (MRI). Intraoperative cryosection analysis revealed no infiltrating tumor cells in the "5-ALA-positive" tissue; however, the non-fluorescent "5-ALA-negative" solid tumor mass showed metastatic malignant melanoma. The postoperative MRI showed a gross total resection of the tumor.

The gross surgical specimens were histologically examined. This included a nodule $(3 \times 2 \times 2 \mathrm{~cm})$ and six largely intact mapping biopsy samples each measuring approximately $0.3-0.4 \mathrm{~cm}$ in diameter. Samples were processed according to standard histopathological techniques (fixation in $4 \%$ buffered formalin; $3-\mu \mathrm{m}$ thick sections; hematoxylin/eosin stain). Light microscopy confirmed that the tumor nodule contained amelanotic malignant melanoma (Fig. 1a). Conversely, all mapping biopsies taken from the peritumoral fluorescent tissue showed edematous cortical and subcortical brain parenchyma with reactive gliosis that was devoid of tumor infiltration (Fig. 1b-d).

To the best of our knowledge, this is the first reported case of a resection procedure for cerebral melanoma metastasis assisted by 5-ALA fluoroscopy. One study has shown that the use of 5-ALA resulted in a significantly improved rate of gross total resection that translated into prolonged progression-free survival [2]. However, the behavior of 5-ALA in cerebral lesions other than malignant glioma, and its value for resection and impact on survival remain undetermined. By analogy to malignant glioma, the 
Fig. 1 a Solid tumor mass (upper two-thirds) composed of plump epithelioid melanocytes largely devoid of pigment granula (magnification $\times 150$ ); relatively sharp circumscription toward peritumoral brain parenchyma (lower third) is observed. $\mathbf{b}_{\mathbf{1}}-\mathbf{b}_{\mathbf{6}}(\times 5)$ Boxes in $\mathbf{b}_{\mathbf{2}}$ and $\mathbf{b}_{\mathbf{3}}$ indicate areas depicted at high magnification $(\times 200)$ in $\mathbf{c}$ and $\mathbf{d}$, respectively. c Detailed view of peritumoral cortex shows reactive astrogliosis with gemistocytic morphology (arrows) $(\times 200)$. d Detailed view of peritumoral white substance reveals spongy to vacuolated aspect due to edema. Reactive astrocytes are highlighted by arrows. All microphotographs represent slides stained with hematoxylin and eosin
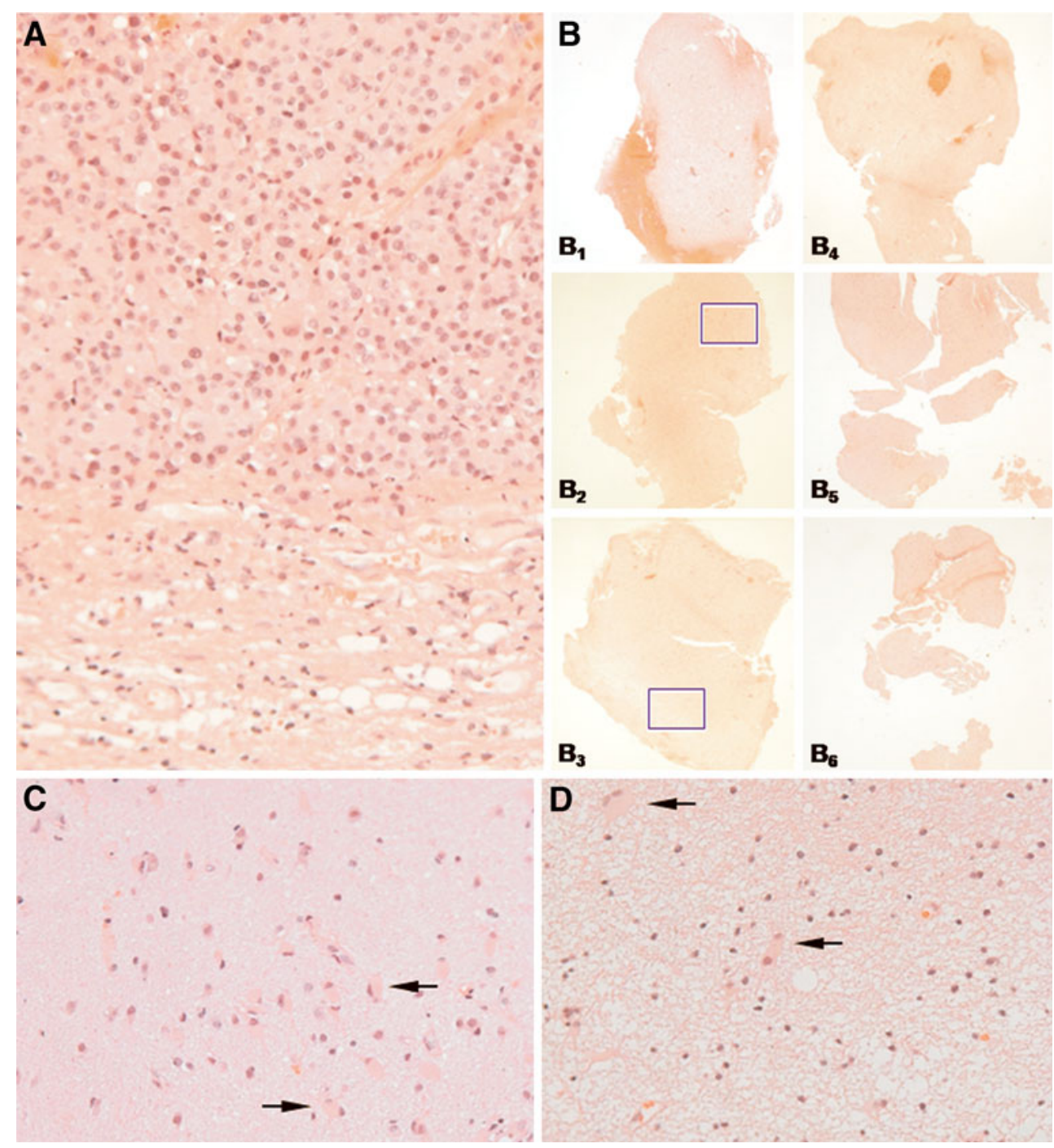

potential benefits - such as a higher rate of complete resections and prolonged local disease control-should be considered for cerebral melanoma metastasis.

An absence of 5-ALA-induced fluorescence has been described in some forms of metastatic deposits [4]. Utsuki et al. [4] reported on a series of peritumoral tissue samples from glioma and cerebral metastasis that had shown vague fluorescence despite the lack of unambiguous infiltration by tumor cells on histology. It has also been suggested that some of the protoporphyrin IX produced in tumor cells may spill outside the tumor boundaries and accumulate in the surrounding tissue [3]. This is an unlikely explanation in our case, since the tumor did not show protoporphyrin accumulation. A vague fluorescence, despite the lack of neoplastic cells, has been attributed to an inflammatory reaction in surgeries for recurrent disease [1]. The pecu- liarity of the present case is the observation of an undescribed constellation of bright fluorescence in the uninvolved perilesional tissue - as opposed to the lack of signal within the tumor itself.

Although the distribution of fluorescence radically departed from an expected pattern, it nevertheless proved of great help in visualizing the tumor-brain interface. It is likely that 5-ALA might evolve into a useful ancillary tool for brain metastasis surgery, provided that consistent fluorescence patterns will have emerged from current and future research.

Acknowledgements The final version of the manuscript was edited by Susan Wieting, Inselspital, Department of Neurosurgery, Publications Office, Bern, Switzerland.

\section{Conflicts of interest None.}




\section{References}

1. Filbeck T, Roessler W, Knuechel R, Straub M, Kiel HJ, Wieland WF (1999) 5-aminolevulinic acid-induced fluorescence endoscopy applied at secondary transurethral resection after conventional resection of primary superficial bladder tumors. Urology 53:77-81

2. Stummer W, Pichlmeier U, Meinel T, Wiestler OD, Zanella F, H-Jr R (2006) Fluorescence-guided surgery with 5-aminolevulinic acid for resection of malignant glioma: a randomised controlled multicentre phase III trial. Lancet Oncol 7:392-401

3. Utsuki S, Miyoshi N, Oka H, Miyajima Y, Shimizu S, Suzuki S, Fujii K (2007) Fluorescence-guided resection of metastatic brain tumors using a 5-aminolevulinic acid-induced protoporphyrin IX: pathological study. Brain Tumor Pathol 24:53-55

4. Utsuki S, Oka H, Sato S, Shimizu S, Suzuki S, Tanizaki Y, Kondo K, Miyajima Y, Fujii K (2007) Histological examination of false positive tissue resection using 5-aminolevulinic acid-induced fluorescence guidance. Neurol Med Chir 47:210-214 